\title{
Opportunistic System for Collaborative Traffic Monitoring Using Existing IEEE 802.11 Networks
}

\author{
José Geraldo Ribeiro Júnior*†, Miguel Elias M. Campista*, and Luís Henrique M. K. Costa* \\ * Grupo de Teleinformática e Automação, UFRJ - COPPE/PEE - DEL/Poli, Rio de Janeiro - RJ - Brazil \\ Email: \{jgrjunior, miguel, luish\}@gta.ufrj.br \\ $\dagger$ CEFET-MG - Campus III - Leopoldina - MG - Brazil \\ Email: \{jgrjunior\}@leopoldina.cefetmg.br
}

\begin{abstract}
Traffic monitoring and control is getting more and more important as the number of vehicles and traffic jams steadily grow. Nevertheless, traffic control is still predominantly done by visual means using strategically placed video cameras. To be more effective, proposals to improve the traffic conditions should consider automated monitoring systems. This work proposes an opportunistic system for collaborative traffic monitoring using available IEEE 802.11 networks. Based on the information received by 802.11 beacon frames, vehicles provide the data needed by a central entity to handle and disseminate information about traffic conditions on urban roads, exploiting readily available network resources. Experiments performed with data from a real public wireless network, in Rio de Janeiro, demonstrate the possibility of obtaining traffic conditions with our proposed traffic monitoring method. The system results are close to GPS traces. Moreover, the proposed system facilitates large-scale adoption since it does not require specific hardware.
\end{abstract}

\section{INTRODUCTION}

At a time when the world is about to reach the mark of almost two billion vehicles in circulation [1], inevitably overloading roads, traffic monitoring and control have become of utmost importance. Big cities, such as Rio de Janeiro, Brazil, have to deal daily with traffic jams as a consequence of car crashes, road repairs or even sudden speed reductions. Another direct consequence of traffic jams is the increased emission of carbon dioxide $\left(\mathrm{CO}_{2}\right)$ and other pollutants in the atmosphere. According to INEA (Rio de Janeiro's Environment Institute), vehicles account for $77 \%$ of the total pollutants emitted into the atmosphere, $25 \%$ to $30 \%$ in a single large avenue, Brazil Avenue [2], an important $58 \mathrm{~km}$ central road in Rio de Janeiro city. Thus, taking preventive actions is a priority.

Proposals to reduce traffic problems require efficient and automated monitoring systems. Nevertheless, the strategy most used in Brazil is still the old-fashioned manually operated video cameras, where all the traffic control is visually done. Some proposals use sensors in cars or along the road [3]-[5], or combine the use of GPS (Global Positioning System) with either GPRS (General Packet Radio Service) [6], or 3G [7][9] or even with IEEE 802.11 [7], [10]-[13]. Whereas sensors are expensive because they are not yet produced in large scale, the use of GPS or $3 \mathrm{G}$ results in battery consumption of portable devices three times larger than IEEE 802.11 [10], [14]. Another problem of $3 \mathrm{G}$ technology, besides the cost to the final user, is the deteriorating quality of service because of the increasing demand, which is making mobile phone companies limit users' maximum rate [15]. The use of GPRS has the advantage of wider coverage because it uses the cellular network, but the upload rate is very low compared with IEEE 802.11 [14].

This paper proposes an opportunistic system for collaborative traffic monitoring using already installed IEEE 802.11 networks. The key idea is based on the popularity of smartphones and on the growth of the number of projects that offer Internet access to wide urban areas using IEEE 802.11. Solely using information derived from beacons of available IEEE 802.11 networks, we infer the location, direction, and speed of vehicles, with no need for new investments or modifications on existing protocols. Client nodes are vital in the process because they are in charge of receiving and filtering data. Afterwards, they send the obtained information to a central unit, which proccesses the received data before sending back consolidated information to users. The user only requires a machine with an IEEE 802.11 interface card to take advantage of the network service.

We evaluate our proposal by collecting and analyzing data on a real scenario. Our experimental results show that it is possible to monitor the traffic conditions based in the received power signal. We evaluated the accuracy of our results compared with control data obtained by GPS. Our results show that the obtained values are very similar and even with a received signal with power as low as $-60 \mathrm{dBm}$ there is a discrepancy of less than 10 meters. A key advantage of our proposal is its capacity to be incrementally deployable since it is not necessary to have $100 \%$ of vehicles sending information.

The remainder of this paper is organized as follows: Section II details our vehicle monitoring system. Section III analyzes experimental results while Section IV concludes the paper and presents topics for future investigation.

\section{Proposed Method}

Traffic monitoring systems do not require a high level of accuracy to find vehicle location on roads [16]. Errors are minimized by predictability of client node movement. Experiments presented in Section III show that a signal received 
with power near $-70 \mathrm{dBm}$ is enough to assess the vehicle location. A signal received at $-60 \mathrm{dBm}$ or higher presents a discrepancy of less than 10 meters. This information was obtained by comparing the position of the strongest signal with the AP (Access Point) physical location in Brazil Avenue. In Figure 1, for example, the signal is stronger on points $B$ and $E$. The speed is calculated using the time taken to travel from $B$ to $E$ and the distance between the two points.

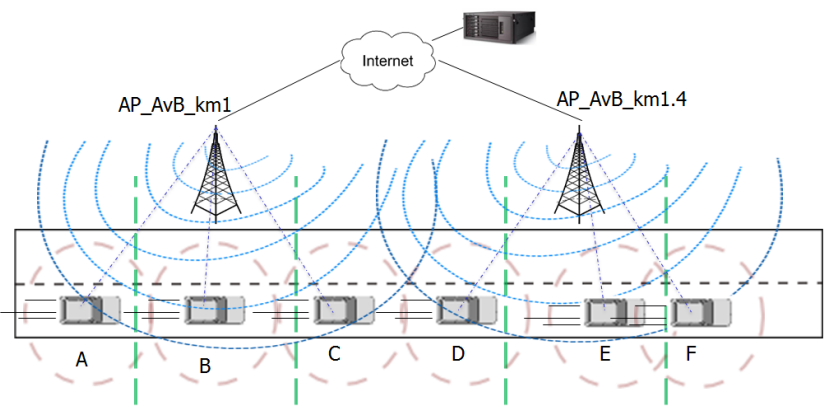

Fig. 1. Example of the scenario used for measurements in Brazil Avenue.

Speed computation is based on beacons periodically sent by APs (by default, the beacon frame is transmitted every $100 \mathrm{~ms}$ ) to obtain all the required information. The beacon is a management frame sent by each AP in the IEEE 802.11 standard. The benefits of using beacons is two-fold: beacons gratuitously offer useful information, such as the ESSID (Extended Service Set ID) of the AP, the MAC address of AP - BSSID (Basic Service Set Identifier), the received signal power, and the time the packet was sent. Second, it is possible to capture beacons in monitor mode (without being associated to an AP), thus not interfering with normal network operation. The combination of network data with previously known information about AP positioning allow the traffic conditions to be obtained in real time. Moreover, our proposed method is collaborative because the client is responsible for receiving, processing, and transmitting the data to be processed by the central unit.

Figure 2 illustrates the architecture of our proposed system. Its main elements: the client node, the access point, and the central unit. It is not necessary to modify any of the elements or protocols between the client and the central unit. These processes will be presented in the Section II-B.

We separate vehicles into four categories: (1) passenger, composed of people carrying communication devices inside vehicles equipped with an IEEE 802.11 interface and executing the client application; (2) preferential, which are vehicles for emergency usage such as police cars, ambulances and, in some cases, buses and taxis; (3) motorcycles; and (4) trucks. This is necessary because preferential vehicles, for example, can present higher speeds in specific scenarios.

When the central unit receives information from at least two vehicles in a stretch of the highway, the system uses the weighted harmonic mean of the received speeds to calculate the condition of that highway stretch. The goal is giving higher weight to more frequent speeds. The weighted harmonic mean of $n$ client nodes speed is the number of nodes divided by the sum of the weight divided of their speeds (Equation 1).

Based on the weighted harmonic mean, the system defines the traffic conditions on the stretch of the highway. From 0 to $19 \mathrm{~km} / \mathrm{h}$, the system indicates that the traffic is SLOW; from 20 to $59 \mathrm{~km} / \mathrm{h}$, the traffic is considered GOOD, and above $60 \mathrm{~km} / \mathrm{h}$, it is considered FAST. For example, in a situation where the central unit receives information from seven vehicles moving at: $20,30,25,40,45,40$, and $50 \mathrm{~km} / \mathrm{h}, n$ is equal 7 and $v_{i}$ is the speed of each vehicle. Thus, the system infers that the traffic condition is GOOD, because the weighted harmonic mean is $32 \mathrm{~km} / \mathrm{h}$.

$$
W H M=\frac{n}{\sum_{i=1}^{n} \frac{w_{i}}{v_{i}}},
$$

where $w_{i}$ and $v_{i}$ are, respectively, the weight and the speed of node $i$, and $v_{i}>0$.

\section{A. Requirements of the Proposed Monitoring System}

Our proposed traffic monitoring system depends on the following conditions (it is worth mentioning that these conditions exist in any scenario similar to Brazil Avenue):

- IEEE 802.11 total or partial coverage on the road;

- periodical dissemination of beacons from APs;

- clients nodes (vehicles);

- APs with known geographical location.

It is not necessary to have constant connection. It is possible to obtain sufficient data even when the APs are distant from each other. The only requirement is a minimal structure to enable users to send the obtained data.

To map the APs position (coordinates) we used three information sources: Google Maps with Street View, data obtained by GPS, and signal obtained with the technique of wardriving [17]. As shown in [18], there are several algorithms to perform this mapping. We consider that the AP is located where the strongest signal is detected because all APs will be, at some time, close to the mobile scanning device. If we know where an AP is on the road (example, km 1.4), we know where (on the road) the vehicle is, associated to it is ( $\mathrm{km} \mathrm{1.4).}$

The next step is to collect information from APs and send it to the central unit, responsible for handling and disseminating data back to users.

Figure 2 presents the system architecture. The process can be divided into five steps, detailed in the following section.

\section{B. System Operation}

Figure 2 illustrates system operation. In step one, when the client application starts, the client IEEE 802.11 interface must be in monitor mode to receive beacons from APs. The goal is to collect beacons of the desired network even before the association process. When the client receives a beacon with known ESSID, it attempts to associate with the AP, using the IEEE 802.11 interface in infrastructure mode. Figure 3 illustrates this process in more detail.

In step two, the client parses only the important information in received data: the AP MAC address, received signal power, 


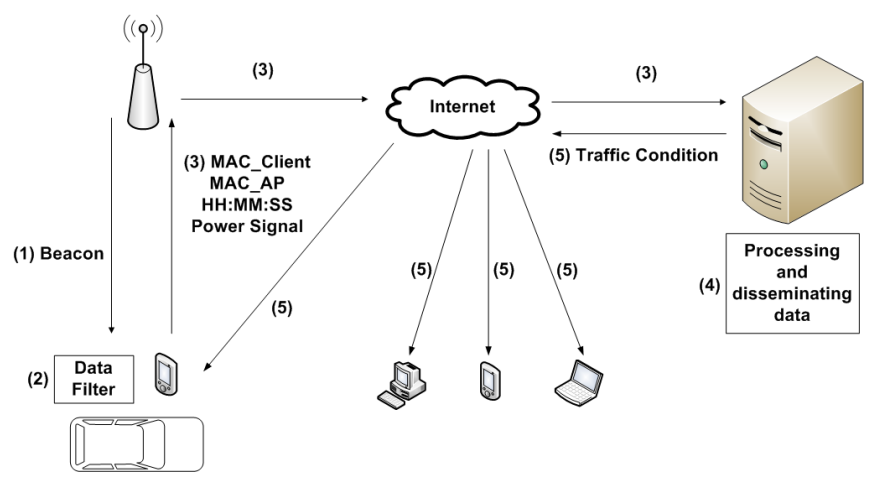

Fig. 2. Proposed system architecture.

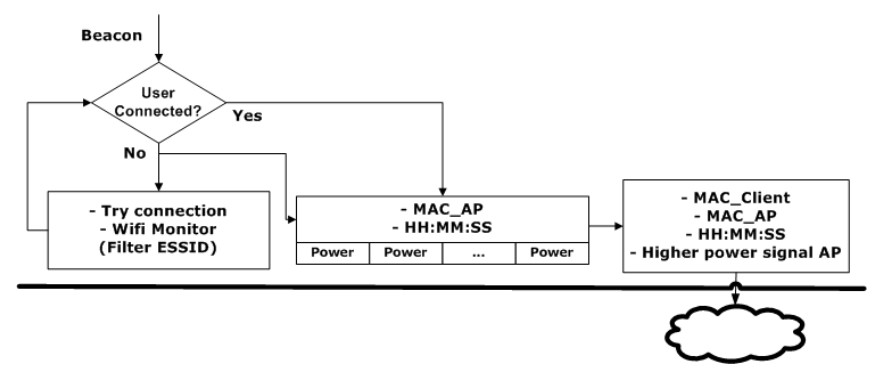

Fig. 3. Client node architecture.

and packet generation time. This data is stored in a sorted structured array, in descending order, where the AP with highest power is in the first position. If the client node is connected, it sends its measurements to the central unit. Otherwise, it stores the measurements until it connects to an AP. In the case of the information being stored locally for a long period, it will no longer be useful for real time monitoring. Nevertheless, it is still sent to the database and, consequently, provides historical data.

In step three,the client sends to the central unit a packet with following the information: the AP MAC and client MAC addresses, received signal power, and time of beacon generation.

Step four is the task of the central unit. Figure 4 presents more details about the central routines. To increase data source confidentiality, only data from registered users is used, other data is discarded. Considering the APs location already inserted into the database, the central unit stores data received from client nodes.

The last step is the dissemination of the traffic information via the Internet. At the moment, we have implemented two types of user interface. The first allows the visualization of the road conditions on a map, using the Google Maps API. For users with equipment without graphic resources, information can be provided in a table format as well, indicating the stretch of the highway, direction, and traffic condition. The information is updated and sent to the client every 30 seconds. Using the records obtained in the last 30 seconds, the central unit calculates the weighted harmonic mean of the speeds in a given stretch of the highway, thus filling the table

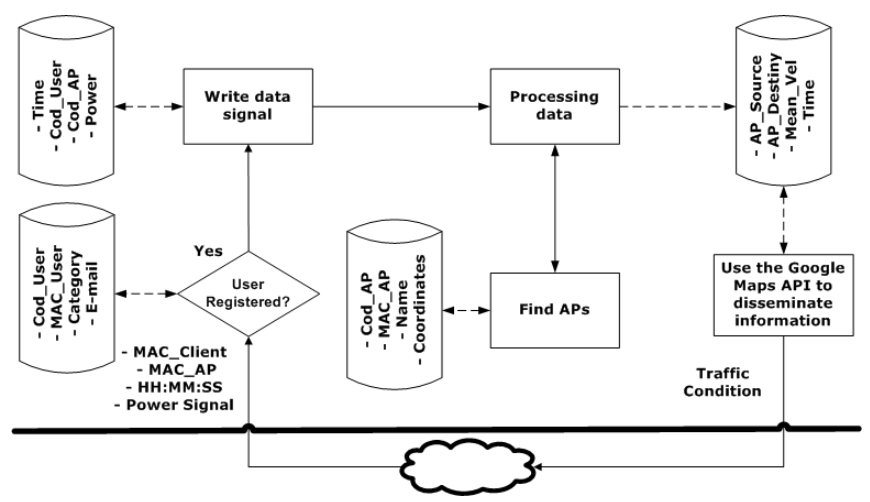

Fig. 4. Central unit architecture.

highwayCondition.

It is the central unit that computes the location and direction of the vehicle in real time. To locate the vehicle on the road, we get the last information sent by the client, indicating which AP it was associated with. To set the vehicle's direction it is necessary to know the MAC address of the last two APs from which the client node sent information to the central unit. It is important to note that the last MAC address is always the AP which the client node is connected to, since it is necessary to be connected to transmit. Thus, this mechanism works even in periods of disconnection since the client stores the information locally until it reconnects.

\section{EXPERIMENTS}

The data used for analysis was obtained from a wireless IEEE $802.11 \mathrm{~b} / \mathrm{g}$ structure designed to cover $58 \mathrm{~km}$ of Brazil Avenue, in Rio de Janeiro. The network connection to the Internet has a bandwidth of $10 \mathrm{Mb} / \mathrm{s}$ which is shared among all users.

First, we map the real location of the APs. According to the project, the APs were deployed every $400 \mathrm{~m}$. Nevertheless, the position of 63 out of the $163 \mathrm{APs}$, distributed along $22.4 \mathrm{~km}$, showed a distance between APs varying from 150 to $400 \mathrm{~m}$. Another problem detected in the avenue mapped stretches is that only 17 APs were working. Figure 5 presents the avenue mapped stretches. The highway stretch highlighted represents the area where the data was collected, corresponding to $6.8 \mathrm{~km}$. All APs were included in the database (coordinates), but only 17 with MAC address.

We collected beacons from these 17 APs using a client executed in a smartphone Nokia N900 running Maemo Linux. We used an Intel Atom N450 laptop with 2 GByte of RAM, Linux, and an internal IEEE 802.11 interface. To assess the precision of our proposed monitoring system we used a GPS model $\mathrm{u}-\mathrm{blox}$ EVK-5H to collect information about time, date, speed, direction, and geographic coordinates. The information was stored locally for later analysis. We collected measurements from $7 \mathrm{~km}$ of the Brazil Avenue, as indicated in Figure 5.

Figures 6 and 7 present the results of the data collected in some Brazil Avenue stretches. Figure 6 plots the signal power 


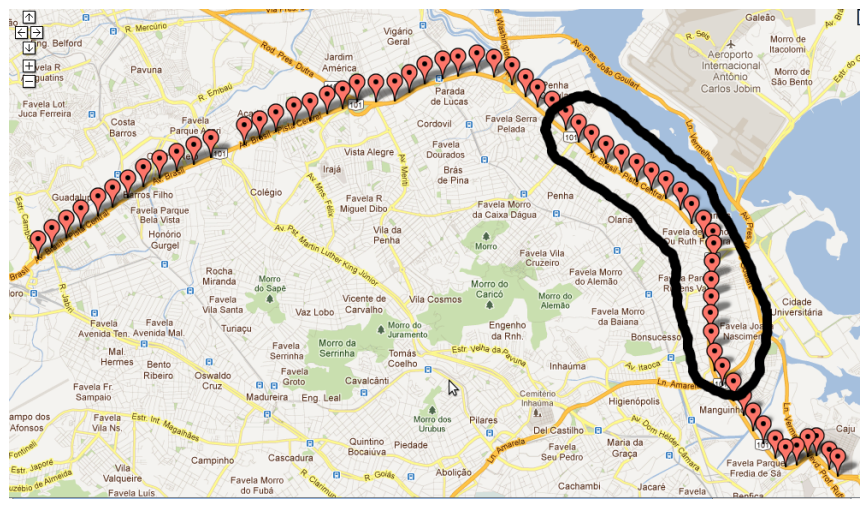

Fig. 5. Brasil Avenue APs mapping.

of the received beacons. The seven APs in the stretch of the highway present the strongest signal around $-60 \mathrm{dBm}$. The speed on the stretch of the highway is calculated using the distance between two APs in function of the time obtained by the subtraction of the instants of the strongest signals. Figure 7 presents the results obtained using GPS, the weighted harmonic mean of the GPS speeds, and the proposed method. The distance between APs varied from $250 \mathrm{~m}$ to $1100 \mathrm{~m}$. Most of the time, the speed has little variation. It is possible to see that the results obtained by the proposed system are very similar to GPS results.

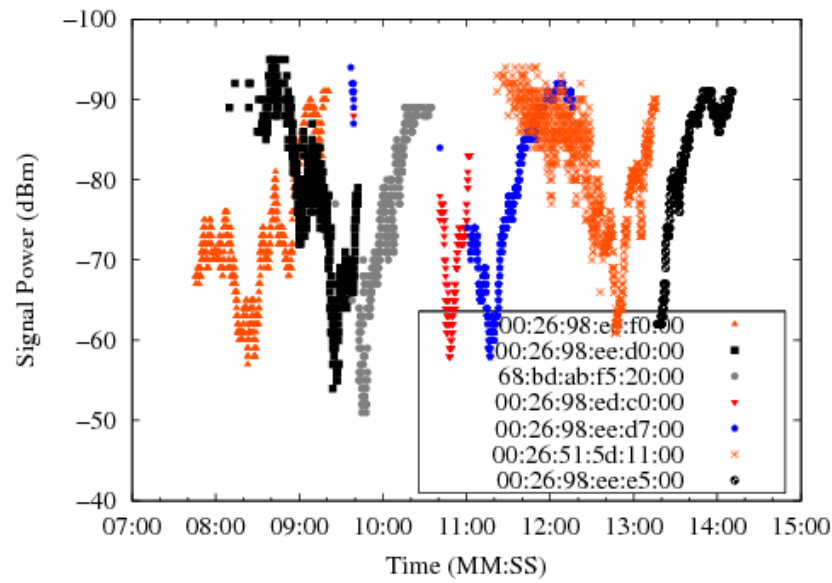

Fig. 6. Signal power received by client node in Stretch 1 of the highway.

Figures 8 and 9 present the results obtained in another stretch of the highway, with $4.2 \mathrm{~km}$. In this stretch of the road we have a wide speed variation. Once again, the result obtained with the proposed method is very close to the weighted harmonic mean of the GPS speeds.

Figure 10 presents an analysis based in six specific situations where we have 15 vehicles on a highway with an exclusive lane for preferential vehicles. The speed of each passenger vehicle is randomly chosen and the speed of the preferential vehicles is $100 \mathrm{~km} / \mathrm{h}$. From the 15 vehicles, there are up to 5 preferential vehicles whereas the others are passenger vehicles. In this experiment, we run 1,000 simulations

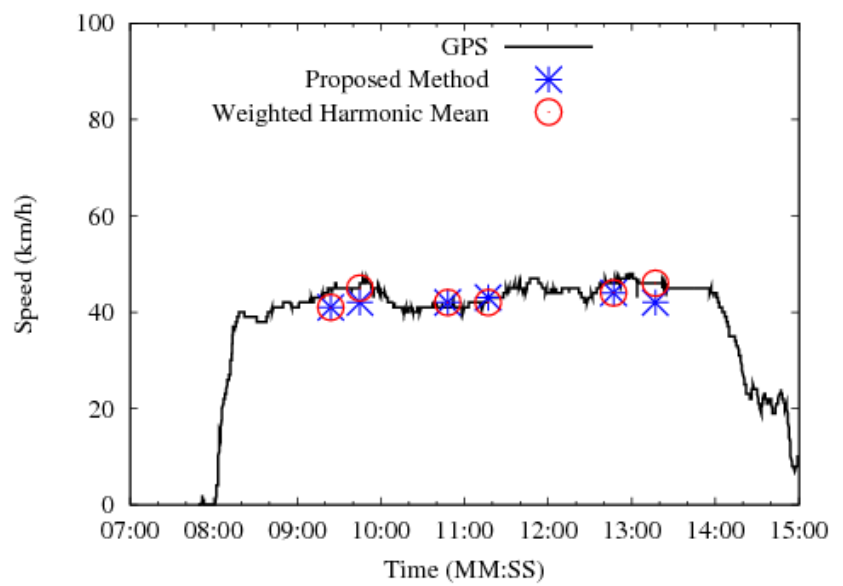

Fig. 7. Results comparison - Stretch 1 of the highway.

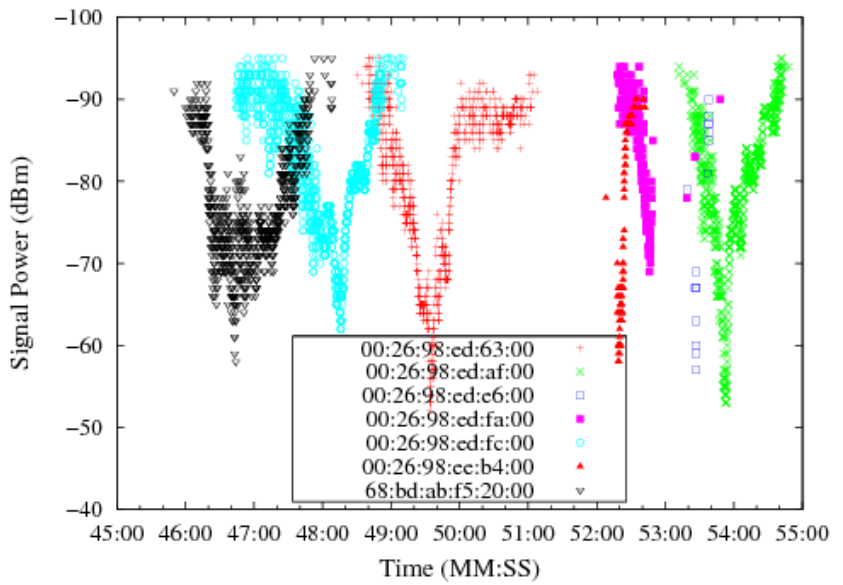

Fig. 8. Signal Power received by client node in Stretch 2 of the highway.

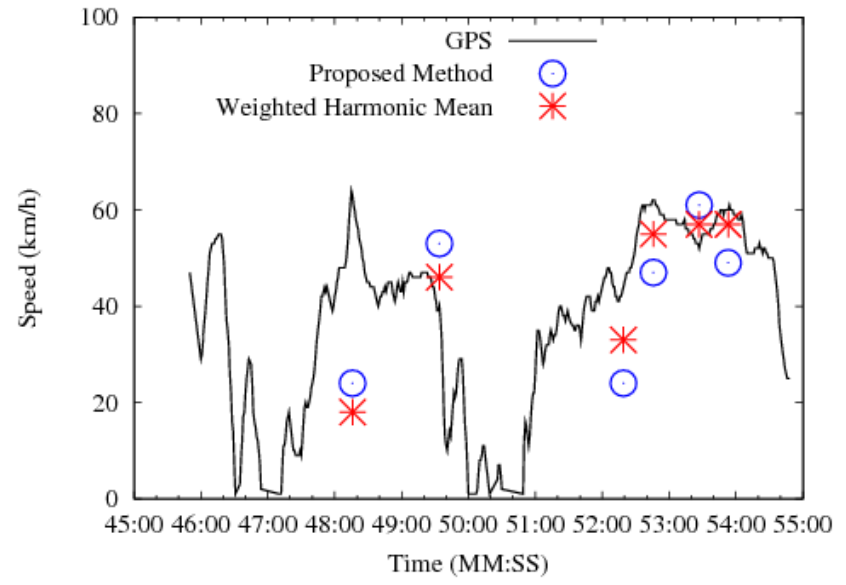

Fig. 9. Results comparison - Stretch 2 of the highway.

for each situation (total 30,000). The goal is to observe the behavior of harmonic mean to define the speed of stretch of the highway in specific situations. In this scenario the harmonic mean is a better choice. Results are promising only because 
in four situations (less than 15\%) we have a relevant number of changes of status and, in these extreme situations, it was necessary to have four preferential vehicles (almost 30\%) to change the status of the road's traffic condition. Nevertheless, the opposite test is demonstrated in [19], where a slow vehicle directly affects the traffic in other lanes. This happens because the traffic flow depends on each roads characteristics, e.g. number of lanes and maximum speed, and on how people drive.

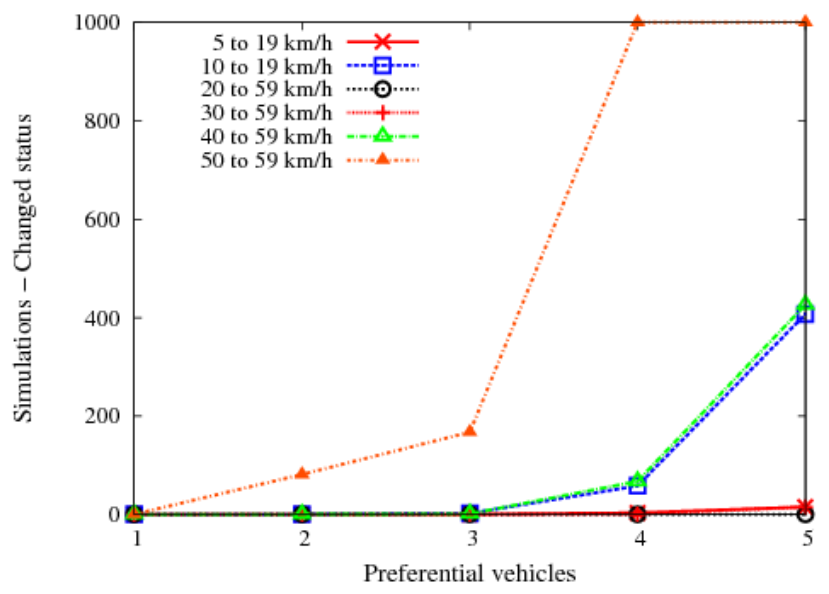

Fig. 10. Preferential vehicles influence.

\section{CONCLUSION}

This work presented an opportunistic system for collaborative traffic monitoring using already installed IEEE 802.11 networks. It is not necessary to change either the network infrastructure or its protocols. Experiments performed with data obtained from a real environment, Brazil Avenue, in Rio de Janeiro, show that a received signal with power from $-60 \mathrm{dBm}$ results in a vehicle positioning error of less than 10 meters.

The proposed monitoring system results are similar to that presented by GPS, but with up to 3 times less battery expenditure on the client node. Our experiments show that the speed of preferential vehicles does not impact on the average velocity of the stretch of the highway. At the same time, the slowest vehicle reflects on all lanes. Nevertheless, the scenarios where the preferential vehicle speed is high enough to modify the traffic status are rare showing the robustness provided by the use of the weighted harmonic mean.

As future work, we will simulate the proposed system behavior with a higher number of client nodes. Another possible direction is to create an alert system to inform the drivers about problems on the road. The alert will be sent via e-mail or message. The idea also allows users to register alerts. Finally, we are going to implement the traffic forecast for specified periods, based on historical data.

\section{ACKNOWLEDGMENTS}

This work was partially funded by CAPES, COFECUB, FAPERJ, CNPq, and FINEP/FUNTTEL.

\section{REFERENCES}

[1] D. Sperling and D. Gordon, Two Billion Cars: Driving Toward Sustainability. Oxford University Press, 2009. [Online]. Available: http://econpapers.repec.org/RePEc:oxp:obooks:9780195376647

[2] I. S. E. Institute, "Annual Report about Air Quality of the Rio de Janeiro State (in Portuguese)." http://www.inea.rj.gov.br/downloads/relatorios/qualidade_ar_2009.pdf, pp. 35-36, 2009, acessed in July 2011.

[3] C. D. T. CDT, "CalTrans," Available at http://www.dot.ca.gov/, 2011, accessed in January 2012.

[4] A. Edelmayer, M. Miranda, and V. Nebehaj, "Cooperative Federated Filtering Approach for Enhanced Position Estimation and Sensor Fault Tolerance in Ad-hoc Vehicle Networks," Intelligent Transport Systems, IET, vol. 4, no. 1, pp. 82 -92, march 2010.

[5] V. Bychkovsky, K. Chen, M. Goraczko, H. Hu, B. Hull, A. Miu, E. Shih, Y. Zhang, H. Balakrishnan, and S. Madden, "The CarTel Mobile Sensor Computing System," in Proceedings of the 4th international conference on Embedded networked sensor systems, ser. SenSys '06. New York, NY, USA: ACM, 2006, pp. 383-384. [Online]. Available: http://doi.acm.org/10.1145/1182807.1182866

[6] C. Smith and N. Watts, "Airsage," Available at http://www.airsage.com/, 2011, accessed in January 2012.

[7] Google, "Google Maps," Available in http://maps.google.com/support, 2011, accessed in January 2012.

[8] Yahoo, "Yahoo Maps," Available in http://maps.yahoo.com/, 2011, accessed in January 2012.

[9] Microsoft, "Microsoft Maps," Available in http://br.bing.com/maps/, 2011, accessed in January 2012.

[10] A. Thiagarajan, L. Ravindranath, K. LaCurts, S. Madden, H. Balakrishnan, S. Toledo, and J. Eriksson, "VTrack: Accurate, Energy-aware Road Traffic Delay Estimation Using Mobile Phones," in Proceedings of the 7th ACM Conference on Embedded Networked Sensor Systems, ser. SenSys '09. New York, NY, USA: ACM, 2009, pp. 85-98. [Online]. Available: http://doi.acm.org/10.1145/1644038.1644048

[11] "Drive-thru Internet," Available in http://www.drive-thru-internet.org, accessed in January 2012.

[12] A. L. Ananda, B. Chen, P. V. S, C. F. Cheong, G. X. Fa, M. Zongyao, L. C. Full, K. S. Praveen, J. Wong, and M. C. Chan, "DTN for Urban Environment," Available in http://mobtorrent.ddns.comp.nus.edu.sg, 2008, accessed in January 2012.

[13] P. R. Group, "A Mobility Testbed - UMass DOME," Available in http://prisms.cs.umass.edu/dome/, accessed in January 2012.

[14] A. Carroll and G. Heiser, "An Analysis of Power Consumption in a Smartphone," in Proceedings of the 2010 USENIX conference on USENIX annual technical conference, ser. USENIXATC'10. Berkeley, CA, USA: USENIX Association, 2010, pp. 21-21. [Online]. Available: http://dl.acm.org/citation.cfm?id=1855840.1855861

[15] A. Balasubramanian, R. Mahajan, and A. Venkataramani, "Augmenting mobile 3G using WiFi," in Proceedings of the 8th international conference on Mobile systems, applications, and services, ser. MobiSys '10. New York, NY, USA: ACM, 2010, pp. 209-222. [Online]. Available: http://doi.acm.org/10.1145/1814433.1814456

[16] A. Boukerche, H. A. B. F. Oliveira, E. F. Nakamura, and A. A. F. Loureiro, "Vehicular Ad Hoc Networks: A New Challenge for Localization-Based Systems," Comput. Commun., vol. 31, pp. 2838 2849, July 2008. [Online]. Available: http://dl.acm.org/citation.cfm?id= 1389585.1389897

[17] B. Issac, S. Jacob, and L. Mohammed, "The Art of War Driving and Security Threats - A Malaysian Case Study," in Networks, 2005. Jointly held with the 2005 IEEE 7th Malaysia International Conference on Communication., 2005 13th IEEE International Conference on, vol. 1, nov. 2005 , p. 6.

[18] Y.-C. Cheng, Y. Chawathe, A. LaMarca, and J. Krumm, "Accuracy characterization for metropolitan-scale Wi-Fi localization," in Proceedings of the 3rd international conference on Mobile systems, applications, and services, ser. MobiSys'05. New York, NY, USA: ACM, 2005, pp. 233-245. [Online]. Available: http://doi.acm.org/10.1145/1067170.1067195

[19] M. Treiber and A. Kesting, "An Open-Source Microscopic Traffic Simulator," Intelligent Transportation Systems Magazine, IEEE, vol. 2, no. 3, pp. $6-13$, fall 2010 . 